\title{
Phase-Controlled Apertures Using Heterodyne Optical Phase-Locked Loops
}

\author{
Naresh Satyan, Wei Liang, Firooz Aflatouni, Amnon Yariv, Anthony Kewitsch, George Rakuljic, and
} Hossein Hashemi

\begin{abstract}
In this letter, we demonstrate the use of an electronic feedback scheme using a voltage controlled oscillator (VCO) to control the optical phase of individual semiconductor lasers (SCLs) phase locked to a common reference laser using heterodyne optical phase-locked loops (OPLLs). The outputs of two external cavity SCLs phase-locked to a common reference laser are coherently combined, and the variation in the relative optical path lengths of the combining beams is corrected by dynamically changing the phase of the offset radio-frequency signal fed into one of the OPLLs by means of a VCO. A stable power combination efficiency of $94 \%$ is achieved. This inherently different method of phase control, i.e., electronic rather than the use of electrooptic crystals, is deemed essential for new applications involving coherent optoelectronics.
\end{abstract}

Index Terms-Coherent optics, optical phase-locked loops (OPLLs), semiconductor laser (SCL) arrays.

\section{INTRODUCTION}

$\mathbf{O}$ PTICAL phase-locked loops (OPLLs) have found applications in microwave photonic systems for coherent optical communications and signal processing [1]. We believe that such phase locking is the basis for a large number of new and significant applications. One of the more interesting of these applications is that of a two-dimensional array of phase-locked semiconductor lasers (SCLs) with arbitrary control of the phase of the individual output beams. Such a control is prerequisite to applications such as coherent power combination, electronic steering of the combined beam, and electronic control of the focusing distance of the combined beam. All of these schemes can be lumped under the title of phase-controlled apertures. The most important aspect of such apertures is the technology employed for the phase control of the individual lasers. We propose a novel electronic feedback scheme using voltage controlled oscillators (VCOs) that enables direct electronic control of the optical phase of an SCL. Electronic feedback con-

Manuscript received November 30, 2007; revised January 15, 2008. This work was supported by the Defense Advanced Research Projects Agency's (DARPA) MTO Office.

N. Satyan is with the Department of Electrical Engineering, California Institute of Technology, Pasadena, CA 911125 USA (e-mail: naresh@ caltech.edu).

W. Liang and A. Yariv are with the Department of Applied Physics, California Institute of Technology, Pasadena, CA 91125 USA (e-mail: liangwei@ caltech. edu; ayariv@caltech.edu).

F. Aflatouni and H. Hashemi are with the Department of Electrical Engineering-Electrophysics, University of Southern California, Los Angeles, CA 90089 USA (e-mail: aflatoun@usc.edu; hosseinh@usc.edu).

A. Kewitsch and G. Rakuljic are with Telaris Inc., Santa Monica, CA 90403 USA (e-mail: kewitsch@telarisinc.com; rakuljic@telarisinc.com).

Color versions of one or more of the figures in this letter are available online at http://ieeexplore.ieee.org.

Digital Object Identifier 10.1109/LPT.2008.922335

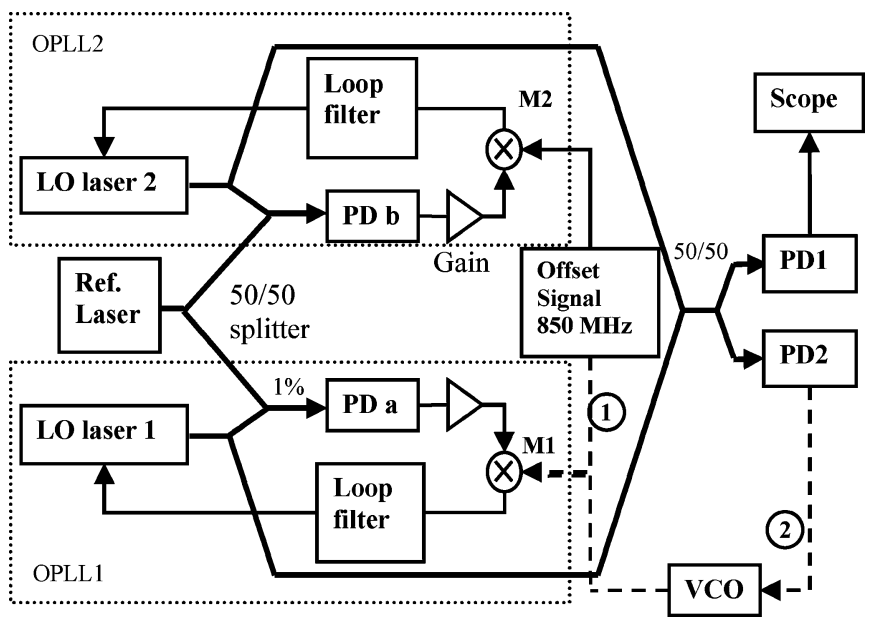

Fig. 1. Experimental setup for the coherent power addition of two SCLs phaselocked to a reference laser using heterodyne OPLLs. With branch 1 connected, a common RF offset signal is fed to both OPLLs. Branch 2 is the closed loop phase compensation scheme using a VCO. PD: photodetectors; M: mixers.

trol eliminates the need for optical feedback or expensive optical components such as optical phase/frequency modulators [2], [3]. Moreover, the VCO functions as an integrating phase shifter, providing a practically unlimited dynamic range for the feedback scheme. In particular, the use of electronic phase control in coherent power combination systems enables efficient, low-cost, high-coherence, high-power sources.

We have recently demonstrated the coherent power combination of commercial SCLs phase-locked to a common reference laser using heterodyne OPLLs [4], [5]. However, the combined power fluctuated "slowly" between its maximum value and zero due to thermal and mechanical variations in the (differential) optical path length traveled by each optical wave. In this letter, we demonstrate the use of the VCO feedback scheme to provide the optical phase shift needed to compensate for the aforementioned drift, achieving a stable power combination efficiency of $94 \%$.

\section{EXPERIMENT}

The basic power combination setup is shown in Fig. 1. Two commercial "slave" local oscillator (LO) SCLs are phase locked to a spectrally stabilized "reference" laser (NP Photonics fiber laser with a 3-dB linewidth of $2.5 \mathrm{kHz}$ ) using a heterodyne OPLL configuration. (For a description of the experiment, see [4].) The reference laser power coupled into each OPLL is about $-3 \mathrm{dBm}$ and could be much lower since the loop gain can be compensated electronically. The two LO (slave) lasers are external cavity SCLs with a nominal output power of $75 \mathrm{~mW}$ 


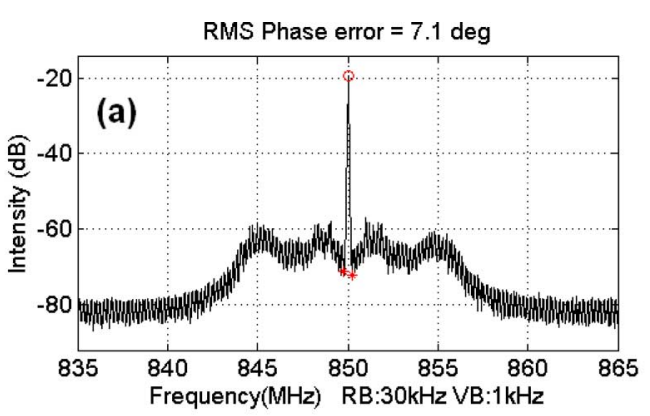

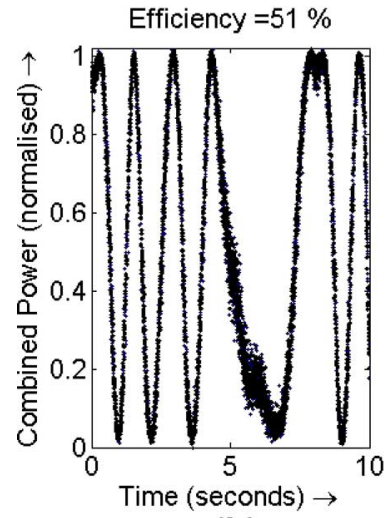

(b)

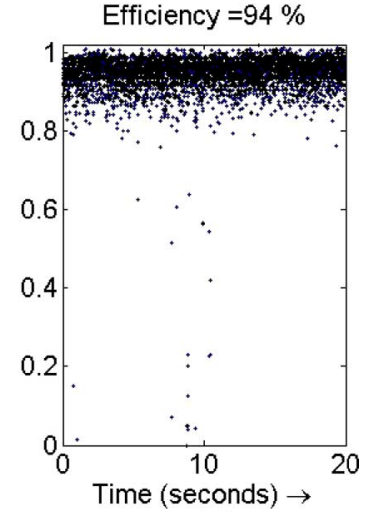

(c)
Fig. 2. (a) Measured power spectrum of the optical beat signal between a single locked LO laser and the reference laser. Variation of the combined power signal as a function of time (b) with a common offset signal and (c) with the VCO compensation scheme.

and a 3-dB linewidth of $0.5 \mathrm{MHz}$. The loop propagation delay in the OPLL is about $5 \mathrm{~ns}$, corresponding to a delay-limited loop bandwidth of $50 \mathrm{MHz}$. The effects of the loop propagation delay and the laser linewidth on the residual phase noise of an OPLL have been investigated in [6], [7]. However, the bandwidth of our OPLL is instead limited to about $3 \mathrm{MHz}$ by the characteristic phase reversal of the FM response of SCLs due to competing thermal and free carrier effects [8]. A loop filter is used to increase the useful loop bandwidth to $5 \mathrm{MHz}$, and the holding range to $+/-200 \mathrm{MHz}$. The measured power spectrum of the optical beat signal between one of the SCLs and the reference laser is shown in Fig. 2(a). Based on the carrier-to-noise ratio calculated from the power spectrum, the root-mean-square phase fluctuation between the LO laser and the reference laser due to the SCL laser noise is about $7^{\circ}$. Initially, branch 1 in Fig. 1 is connected, so that a common RF offset signal is fed to both OPLLs. A variable attenuator is used in one of the inputs of the beam combining coupler to match the input power levels, and PM fiber is used to match the polarizations. The experimental variation of the combined power signal with time is shown in Fig. 2(b). The variation in the differential optical length traversed by the two laser output signals causes the combined power signal to exhibit a slow drift between the maximum and zero, as in Fig. 2(b).

One of the advantages of the heterodyne OPLL scheme is the ability to control the optical phase of the slave SCLs by varying the phase of the RF offset signal, since the optical phase tracks on a $1: 1$ basis the phase of the offset signal. This can be leveraged to compensate for the slow variation in the optical lengths

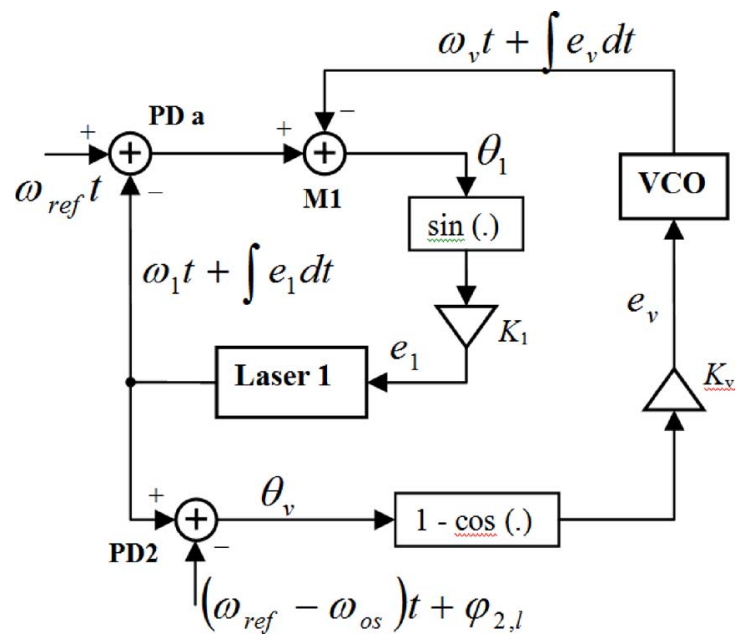

Fig. 3. Steady state phase model of the VCO combination scheme. The LO laser 2 is locked to the reference laser in OPLL2, and is not shown here.

traversed by the two SCL optical waves. This is achieved by feeding back one of the outputs of the $2 \times 2$ beam combiner (PD2) into the VCO supplying the RF offset signal in OPLL1, i.e., by connecting branch 2 in Fig. 1 to close the loop. The VCO thus also acts as an integrating phase shifter with unlimited phase range. This eliminates the disadvantage caused by the limited dynamic range of optical or electronic phase modulators, where phase-unwrapping circuitry is required to prevent cycle-slipping when the noise amplitude is high.

\section{THEORY, RESULTS, AND DISCUSSION}

The steady state phase model of the VCO combination scheme is shown in Fig. 3. The LO laser 2 is locked to the reference laser (of frequency $\omega_{\text {ref }}$ ) at a frequency offset of $\omega_{\mathrm{os}}$, and has a residual phase noise of $\varphi_{2, l}(t) . \omega_{1}$ and $\omega_{v}$ are the free running frequencies of the LO laser 1 and the VCO, respectively. $K_{1}$ is the OPLL gain given by the product of the gains of the photodetector PDa, mixer and the loop filter, and the FM responsivity of the laser. Similarly, $K_{v}$ is the net gain in the VCO branch given by the product of the gain of the photodetector PD2 and the FM responsivity of the VCO. Referring to Fig. 3, $\theta_{1}(t)$ and $\theta_{v}(t)$ are given by

$$
\begin{aligned}
\theta_{1}(t)= & \omega_{\mathrm{ref}} t-\left(\omega_{1} t+\int_{-\infty}^{t} K_{1} \sin \theta_{1}(t) d t\right) \\
& -\left(\omega_{v} t+\int_{-\infty}^{t} K_{v}\left(1-\cos \theta_{v}(t)\right) d t\right) \\
\theta_{v}(t)= & \left(\omega_{1} t+\int_{-\infty}^{t} K_{1} \sin \theta_{1}(t) d t\right) \\
& -\left(\left(\omega_{\text {ref }}-\omega_{\mathrm{os}}\right) t+\phi_{2, l}\right) .
\end{aligned}
$$

The steady state operating point of the system is obtained by setting the time derivatives of the mixer (M1) and photodetector (PD2) outputs $\theta_{1}(t)$ and $\theta_{v}(t)$ to zero in (1) and (2), giving

$$
\begin{aligned}
& \theta_{1 s}=\sin ^{-1}\left(\frac{\omega_{\mathrm{ref}}-\omega_{1}-\omega_{\mathrm{os}}}{K_{1}}\right) \\
& \theta_{v s}=\cos ^{-1}\left(1-\frac{\omega_{\mathrm{os}}-\omega_{v}}{K_{v}}\right) .
\end{aligned}
$$


If $P_{0}$ is the power of each combining optical wave, the total combined power detected at the photodetector PD1 is

$$
P=P_{0}\left(1+\cos \theta_{v}\right)
$$

For maximum power combination efficiency, $\theta_{v s}$ should be as close to zero as possible. From (4), the power combination efficiency can be tuned by varying $\omega_{v}$, the free running frequency of the VCO. One hundred percent efficiency is achieved when the VCO free running frequency is made equal to the offset signal frequency $\omega_{\text {os }}$. However, there is a trade-off between combination efficiency and frequency of cycle slips, as can be seen from (4). As $\theta_{v s}$ approaches zero, frequency jittering of the VCO can cause the quantity $\omega_{\text {os }}-\omega_{v}$ to take a negative value, in which case there is no solution to (4) and the VCO loop loses lock. Therefore, the frequency noise of the free running VCO compared to the loop gain $K_{v}$ limits the minimum value that $\theta_{v s}$ can take. Further, the residual phase noise in the loop effectively changes the VCO frequency and can throw the loop out of lock.

The VCO loop bandwidth and the gain $K_{v}$ are limited by the delay in the fiber [6], so that $K_{v} \ll K_{1}$. When this condition is satisfied, the overall system can be regarded as the combination of two closed loop systems-a "fast" control loop (the OPLL) that tracks the phase noise of the LO laser 1 relative to the reference laser within the OPLL bandwidth, and a "slow" VCO loop that corrects for the phase noise arising due to the slow variations in the optical path length. Fig. 2(c) shows the combined power using the $\mathrm{VCO}$ feedback scheme, and demonstrates the high combination efficiency achievable by this scheme. The combined power is held constant with a power combination efficiency of about $94 \%$. This efficiency is mainly limited by the nonzero value of $\theta_{v s}$, and can be further improved by the use of a cleaner VCO and lag-lead filters in the VCO feedback path to shape the loop transfer function and increase the dc gain.

\section{CONCLUSION}

We have demonstrated the use of an electronic feedback scheme using a VCO for the coherent power combination of two commercial external cavity SCLs phase-locked to a common reference signal. The feedback scheme has been used to compensate for fluctuations in the optical path lengths of the combining optical waves, and a combination efficiency of $94 \%$ has been achieved. The scheme is capable of compensating for additional phase noise sources such as fiber amplifiers, and is scalable to larger systems involving the coherent power combination of a large number of SCLs locked to a single reference laser.

\section{REFERENCES}

[1] L. N. Langley, M. D. Elkin, C. Edge, M. J. Wale, U. Gliese, X. Huang, and A. J. Seeds, "Packaged semiconductor laser optical phase-locked loop (OPLL) for photonic generation, processing and transmission of microwave signals," IEEE Trans. Microw. Theory Tech., vol. 47, no. 7, pp. 1257-1264, Jul. 1999.

[2] S. J. Augst, T. Y. Fan, and A. Sanchez, "Coherent beam combining and phase noise measurements of ytterbium fiber amplifiers," Opt. Lett., vol. 29, no. 5, pp. 474-476, 2004.

[3] L. Bartlett-Berger, U. Brauch, A. Giesen, and H. Opower, "Power-scalable system of phase-locked single-mode diode lasers," Appl. Opt., vol. 38 , no. 27 , pp. 5752-5760, 1999.

[4] W. Liang, A. Yariv, A. Kewitsch, and G. Rakuljic, "Coherent combining of the output of two semiconductor lasers using optical phaselock loops," Opt. Lett., vol. 32, pp. 370-372, 2007.

[5] W. Liang, N. Satyan, A. Yariv, A. Kewitsch, G. Rakuljic, F. Aflatouni, H. Hashemi, and J. Ungar, "Coherent power combination of two master-oscillator-power-amplifier (MOPA) semiconductor lasers using optical phase lock loops," Opt. Express, vol. 15, pp. 3201-3205, 2007.

[6] M. Grant, W. Michie, and M. Fletcher, "The performance of optical phase-locked loops in the presence of nonnegligible loop propagation delay," J. Lightw. Technol., vol. 5, no. 4, pp. 592-597, Apr. 1987.

[7] R. T. Ramos and A. J. Seeds, "Delay, linewidth and bandwidth limitations in optical phase-locked loop design," Electron. Lett., vol. 26, no. 6, pp. 389-391, Mar. 1990

[8] P. Correc, O. Girard, and I. F. de Faria Jr., "On the thermal contribution to the FM response of DFB lasers: Theory and experiment," IEEE $J$. Quantum Electron., vol. 30, no. 11, pp. 2485-2489, Nov. 1994. 